\title{
We Cannot Solve a Problem, Using the Same Mentality Which We Have Created Them
}

\author{
Dmitrieva Elena Germanovna* \\ A Clinical Pharmacist, Cand. Biol. Sci. on a speciality clinical pharmacology, Russia \\ *Corresponding author: Dmitrieva Elena Germanovna, a Clinical Pharmacist, Cand. Biol. Sci. on a speciality clinical pharmacology, Sakko's street, \\ House 3, Room 51, Town Ivanovo, Russia
}

Submission: 監 :November 12, 2018; Published: 眥 November 26, 2018

\section{Opinion}

A. We Cannot Solve a Problem, Using the Same Mentality Which We Have Created Them-A.Einstein

B. Competent treatment infections at Children and Adults Alternative of vaccination.

C. Opinion of the expert - of the Expert-clinical of the Pharmacist.

Vaccination (from a Latin word - a vacca-cow or an inoculation introduction of an antigene material on purpose to cause immunity to illness which will prevent infection or will weaken its negative consequences.

It not preventive maintenance of illnesses as doctors impose, for example, in Russia, and infection with infectious disease. It can be bacteria or viruses. By the way, $80 \%$ of all virus pneumonia at children till 5 years have the virus nature.

The analysis of by-effects from vaccines since 1983 has shown, that till 3 years it is impossible in general it is impossible to do any vaccines. I not the ardent opponent of vaccination, but I consider, that it is necessary to do all on an epidemic situation and that not by all.

I know children who at direct contact to any contagious did not fall ill with an infection and when put vaccines - fell ill seriously. So it is necessary to approach to all individually. Then from medicine there will be a sense for health of children and adults. It is impossible to heal millions. By the way, adults after 50 years too cannot put inoculations as they «do not work».

For years of compulsory vaccination, it was necessary to see and mourn over children much. All time I say to all people, that I am compelled to go on cemeteries and to mourn over innocent children.

My opinion is that, that problems need to be solved in process of their receipt when infection has already occurred. I understand, what many will tell, and how preventive maintenance? But it's not preventive maintenance, and infection. Preventive maintenance of illnesses is when, for example, immunity lift, instead of deform it or destroy completely though, for today these barbarous methods exist and in oncology. And I am ready to give and alternative to a children's cancer.

In Russia in many establishments is not present immunological laboratories, there is no possibility to make analyses on presence of antibodies in blood after vaccination, there is no possibility to block toxic actions of vaccines and many other things. To take temperature before a vaccine is an attribute of the last century.

And, for example, the vaccine from a natural smallpox has been justified not to disfigure the person, an inoculation did on hips. But, when this «therapy» began to be applied on many infections, and on what can be avoided, conducting a healthy way of life vaccination sees strange and dangerous. I know many doctors whom does not do vaccines to itself, to children and grandsons, and put experiments on others. And, of by-effects dies to $2 \%$ of children, and these are huge figures in population of children to 3 summer age. In Russia the statistics of such cases do not conduct, and such cases disappear. In medical cards and case records write other diagnoses. In Russia doctors do not love children and the people. There are no children of strangers, all of them ours.

Many sane doctors to Russia, fortunately the such are available, recognise, that pandemics of infections depend on quantity of the put vaccines. Why, tell you? And all business again in individuality of each small and adult person. One this vaccine is transferred normally, by another with by-effects, and the third will be lost. There are also other outcomes of situations when itself is not ill, but becomes the carrier of infections and extends in surrounding space of an infection, from which as from a vaccine, others catch.

More professional language it is possible to tell, that exist and for today are described strains infectious diseases, syndromes imprinting's and infection strengthenings are recognised. These are terrible reactions in the world-infection with infections. And these reactions should be stopped. It is alternative of parents of children. They mark for health of children. In Russia health of children is dumped on a payoff of parents vaccination doctors violently, a deceit and another manipulations, thus, impose. If a precept of the doctor - do not do much harm, why harm? 
It is necessary to treat when it is necessary to treat, when the person was ill, but to treat competently. It is impossible to vaccinate all. Each person is especially individual. That is possible for one, is categorically counter-indicative to another.

I wish to help Children, as to the most defenceless beings on the Earth. As especially terrible vaccines are considered - BCG and a hepatitis B - especially their combination. The in itself vaccine for tuberculosis infection - kills immunity of the child that is then fraught with serious consequences in health. The combination of these two vaccines - gives a nejro-infection in white substance of a brain that does generations of children by patients. There are such researches. All vaccines get into a brain of the child and a consequence so are not predicted. It not only a meningitis, but also imbecility. The remote consequences of vaccines is a multiple sclerosis at young women and lateral amyotrophic a sclerosis of young men. It and strengthening infectious atherogenesis, and this accelerated ageing, increase libido at children, and an early climax at adults. Maniacs, pedophiles - unless not from changes in brain structure undertake?

Vaccine AKDS - amazes nervous system. Other vaccines have weight of by-effects which are held back. Each illness even with an easy current gives weight of complications, forming the person margenal's.

After inoculations there is no proof lifelong immunity from infectious diseases. And them it is possible to be ill many times. Unless it can arrange?
I understand, that many will begin to groan, that a pier, there are no exact data. And what are necessary data? If suffers and dies though one child it is not necessary for it. Children should be healthy and live happily.

From vaccines dies more than of infections, more often, not cured. If in the beginning and the middle of 20 centuries there were no such methods of treatment now is. It is necessary to refuse from old, as unnecessary stuff in the house. Also it is necessary to search for other methods of competent treatment not to harm to children!

It is ready to describe all collateral and toxic effects of the vaccines, competent treatment of all infections, both at children, and at adults. I am ready to give pure spring water for Clinical Pharmacists, who always nearby at bed of patients and who can protect them!

For today I already have described competent treatment without vaccines and even some blocators vaccines when they are fraught with toxic effects. These are such infections in the childhood - measles, rubella, Staphylococcus aureus, blue pus bacillus. Is ready to give alternative treatment on other infections.

But I require financing on these purposes, as this work very heavy, unbearable. I should have time to make all for children and to protect them as much as possible! My long-term clinical experience has proved, that only I can make it if nobody could make it earlier though, in Europe many have doubted efficiency of any vaccination.

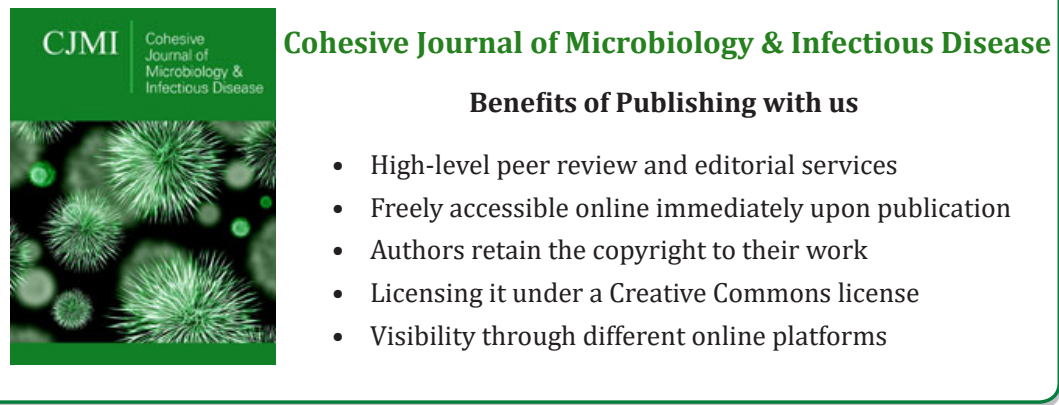

